OPEN ACCESS

Edited by:

Stuart Brian Hooper, Monash University, Australia

Reviewed by:

Britt Nakstad,

University of Oslo, Norway

Liam Mahoney,

Southmead Hospital, United Kingdom

*Correspondence:

Georg M. Schmölzer georg.schmoelzer@me.com

Specialty section:

This article was submitted to

Neonatology,

a section of the journa

Frontiers in Pediatrics

Received: 13 December 2020 Accepted: 25 January 2021

Published: 12 February 2021

Citation:

Wagner $M$, Cheung $P-Y$, Yaskina $M$, Lee $T-F$, Vieth VA, O'Reilly $M$ and Schmölzer GM (2021) Return of Spontaneous Circulation Depends on Cardiac Rhythm During Neonatal Cardiac Arrest in Asphyxiated Newborn Animals.

Front. Pediatr. 9:641132.

doi: 10.3389/fped.2021.641132

\section{Return of Spontaneous Circulation Depends on Cardiac Rhythm During Neonatal Cardiac Arrest in Asphyxiated Newborn Animals}

\author{
Michael Wagner 1,2, Po-Yin Cheung 2,3, Maryna Yaskina ${ }^{3}$, Tze-Fun Lee ${ }^{2}$, Vanessa A. Vieth ${ }^{1}$, \\ Megan O'Reilly ${ }^{2,3}$ and Georg M. Schmölzer ${ }^{2,3,4 *}$ \\ ${ }^{1}$ Division of Neonatology, Pediatric Intensive Care and Neuropediatrics, Department of Pediatrics, Comprehensive Center for \\ Pediatrics, Medical University of Vienna, Vienna, Austria, ${ }^{2}$ Neonatal Research Unit, Centre for the Studies of Asphyxia and \\ Resuscitation, Royal Alexandra Hospital, Edmonton, AB, Canada, ${ }^{3}$ Department of Pediatrics, University of Alberta, \\ Edmonton, AB, Canada, ${ }^{4}$ Division of Neonatology, Department of Pediatrics, Medical University Graz, Graz, Austria
}

Objective: Pulseless electrical activity (PEA) occurs in asphyxiated newborn piglets and infants. We aimed to examine whether different cardiac rhythms (asystole, bradycardia, $\mathrm{PEA}$ ) affects the resuscitation outcomes during continuous chest compressions (CC) during sustained inflations (CC+SI).

Design: This study is a secondary analysis of four previous randomized controlled animal trials that compared CC+SI with different CC rate (90 or 120/min), SI duration (20 or $60 \mathrm{~s})$, peak inflation pressure $\left(10,20\right.$, or $\left.30 \mathrm{cmH}_{2} \mathrm{O}\right)$, and oxygen concentration $(18,21$, or $100 \%)$.

Setting and Subjects: Sixty-six newborn mixed breed piglets (1-3 days of age, weight $1.7-2.4 \mathrm{~kg}$ ) were obtained on the day of experimentation from the University Swine Research Technology Center.

Interventions: In all four studies, piglets were randomized into intervention or sham. Piglets randomized to "intervention" underwent both hypoxia and asphyxia, whereas, piglets randomized to "sham" received the same surgical protocol, stabilization, and equivalent experimental periods without hypoxia and asphyxia.

Measurements: To compare differences in asphyxiation time, time to return of spontaneous circulation (ROSC), hemodynamics, and survival rate in newborn piglets with asystole, bradycardia or PEA.

Main Results: Piglets with PEA $(n=29)$ and asystole $(n=13)$ had a significantly longer asphyxiation time and time to ROSC vs. bradycardia $(n=24)$. Survival rates were similar between all groups. Compared to their baseline, mean arterial pressure and carotid blood flow were significantly lower $4 \mathrm{~h}$ after resuscitation in all groups, while being significantly higher in the bradycardia group.

Conclusion: This study indicates that cardiac rhythm before resuscitation influences the time to ROSC and hemodynamic recovery after ROSC.

Keywords: cardiac rhythm, chest compressions, neonatology, resuscitation, sustained inflation 


\section{INTRODUCTION}

At birth, the clinical team assesses the infant's heart rate (HR) to guide intervention during neonatal resuscitation (1). If HR is $<60 / \mathrm{min}$, chest compressions (CC) must be started (1) Bradycardia and asystole were believed to be the most common cardiac arrest rhythms in newborn infants (2). However, there is a lack of data what HR cut-off to start CC should be used in neonatal patients with bradycardia secondary to asphyxia. Most recently, studies have reported that pulseless electrical activity (PEA) is commonly observed in asphyxiated newborn piglets and infants (3-6). PEA displays an organized cardia rhythm on the electrocardiogram (ECG) without cardiac output or essential blood flow, and is mostly caused by hypoxia and hypovolemia (4).

If a newborn requires chest compression, the neonatal resuscitation guideline recommends a 3:1 Compression:Ventilation $(\mathrm{C}: \mathrm{V})$ ratio $(1,7,8)$. An alternative approach of neonatal chest compression, which combines CC during continuous sustained inflation (SI) (providing constant high airway pressure during $\mathrm{CC}=\mathrm{CC}+\mathrm{SI}$ ) has been described by our group (9-13). The CC+SI approach significantly improved systemic and regional hemodynamics, tidal volume delivery, minute ventilation, and time to return of spontaneous circulation (ROSC) compared to 3:1 C:V in neonatal piglets (14).

During adult cardiac arrest, the presenting cardiac rhythm is most often (81\%) non-shockable (i.e., asystole or PEA) (15). In pediatric patients, the presenting cardiac arrest rhythms are asystole (58\%), bradycardia (6\%), PEA (15\%), ventricular fibrillation or tachycardia (8\%), and unknown rhythms (13\%) (16). In comparison, there is a lack of data about the presenting cardiac arrest rhythm in newborns in the delivery room. Kumar et al. (17) reported 50 infants with asystole and 160 infants with bradycardia over a 16-year period (17). Overall, the time to return of spontaneous circulation (ROSC) was 12.5 min compared to $7 \mathrm{~min}$ in infants with asystole vs. bradycardia. There is limited evidence about outcomes of PEA in newborns. Therefore, we aimed to examine the outcomes of neonatal resuscitation and recovery depending on the presenting cardiac rhythm (i.e., bradycardia vs. PEA vs. asystole) in asphyxiated newborn piglets.

\section{MATERIALS AND METHODS}

This is a secondary analysis of our four previous randomized controlled animal trials, which examined CC+SI. Only data from piglets resuscitated with $\mathrm{CC}+\mathrm{SI}$ were examined.

To optimize the effectiveness of $\mathrm{CC}+\mathrm{SI}$ in resuscitation, series of experiments were carried out to examine whether the outcome can be improved by alternating the CC rate (90 or 120/min) (Study 1)(11), SI duration (20 or 60 s) (Study 2) (18), inflation pressure (10, 20, or $30 \mathrm{cmH}_{2} \mathrm{O}$ ) (Study 3) (19) or oxygen concentration (18, 21, or 100\%) (Study 4) (20). As no difference was observed among groups with various interventions and

Abbreviations: HR, heart rate; CC, chest compressions; PEA, pulseless electrical activity; ECG, electrocardiogram; C:V, Compression: Ventilation; SI, sustained inflation; ROSC, return of spontaneous circulation; CABF, carotid arterial blood flow. the experimental protocols as well as piglets breed were also similar within all these studies, all data were combined for secondary analyses.

Sixty-six newborn mixed breed piglets (1-3 days of age, weighing $1.7-2.4 \mathrm{~kg}$ ) were obtained on the day of experimentation from the University Swine Research Technology Center. All experiments were conducted in accordance with the guidelines and approval of the Animal Care and Use Committee (Health Sciences), University of Alberta (AUP00001764, AUP00002151, and AUP00002651), presented according to the ARRIVE guidelines (21) and registered at preclinicaltrials.eu (PCTE0000138). A graphical display of the study protocol is presented in Figure 1.

\section{Animal Preparation}

Following the induction of anesthesia using isoflurane, piglets were intubated via a tracheostomy, and pressure-controlled ventilation (Sechrist infant ventilator model IV-100; Anaheim, USA for series 1 and Acutronic Fabian HFO; Hirzel, Switzerland for series 2-4) was commenced at a respiratory rate of 16-20 breaths/min and pressure of $20 / 5 \mathrm{cmH}_{2} \mathrm{O}$. Oxygen saturation was kept within 90-100\%, glucose level and hydration were maintained with an intravenous infusion of 5\% dextrose at 10 $\mathrm{mL} / \mathrm{kg} / \mathrm{hr}$. During the experiment, anesthesia was maintained with intravenous propofol $(5-10 \mathrm{mg} / \mathrm{kg} / \mathrm{h})$ and morphine $(0.1$ $\mathrm{mg} / \mathrm{kg} / \mathrm{h})$. Additional doses of propofol $(1-2 \mathrm{mg} / \mathrm{kg})$ and morphine $(0.05-0.1 \mathrm{mg} / \mathrm{kg})$ were given as needed. Only in series 1, pancuronium $(0.1-0.2 \mathrm{mg} / \mathrm{kg})$ was used for controlling the arterial $\mathrm{CO}_{2}$ level. The piglet's body temperature was maintained at $38.5-39.5^{\circ} \mathrm{C}$ using an overhead warmer and a heating pad $(9,14,22)$.

\section{Hemodynamic Parameters}

A 5-French Argyle ${ }^{\circledR}$ (Klein-Baker Medical Inc., San Antonio, TX) double-lumen catheter was inserted via the right femoral vein for administration of fluids and medications. A 5-French Argyle ${ }^{\circledR}$ single-lumen catheter was inserted above the right renal artery via the femoral artery for continuous arterial blood pressure monitoring in addition to arterial blood gas measurements. The right common carotid artery was exposed and encircled with a real-time ultrasonic flow probe $(2 \mathrm{~mm}$; Transonic Systems Inc., Ithica, NY) to measure carotid arterial blood flow (CABF). Piglets were placed in supine position and allowed to recover from surgical instrumentation until baseline hemodynamic measures were stable (minimum of $1 \mathrm{~h})$. Ventilator rate was adjusted to keep the partial arterial $\mathrm{CO}_{2}$ pressure between 35 and $45 \mathrm{mmHg}$, as determined by periodic arterial blood gas analysis. Baseline blood gas values were obtained after stabilization and just before hypoxia. Mean systemic arterial pressure, HR, and percutaneous oxygen saturation were continuously measured and recorded throughout the experiment with a Hewlett Packard 78833B monitor (Hewlett Packard Co., Palo Alto, CA).

\section{Cerebral Perfusion}

Cerebral oxygenation was measured using the Invos Cerebral/Somatic Oximeter Monitor (Invos 5100, Somanetics 


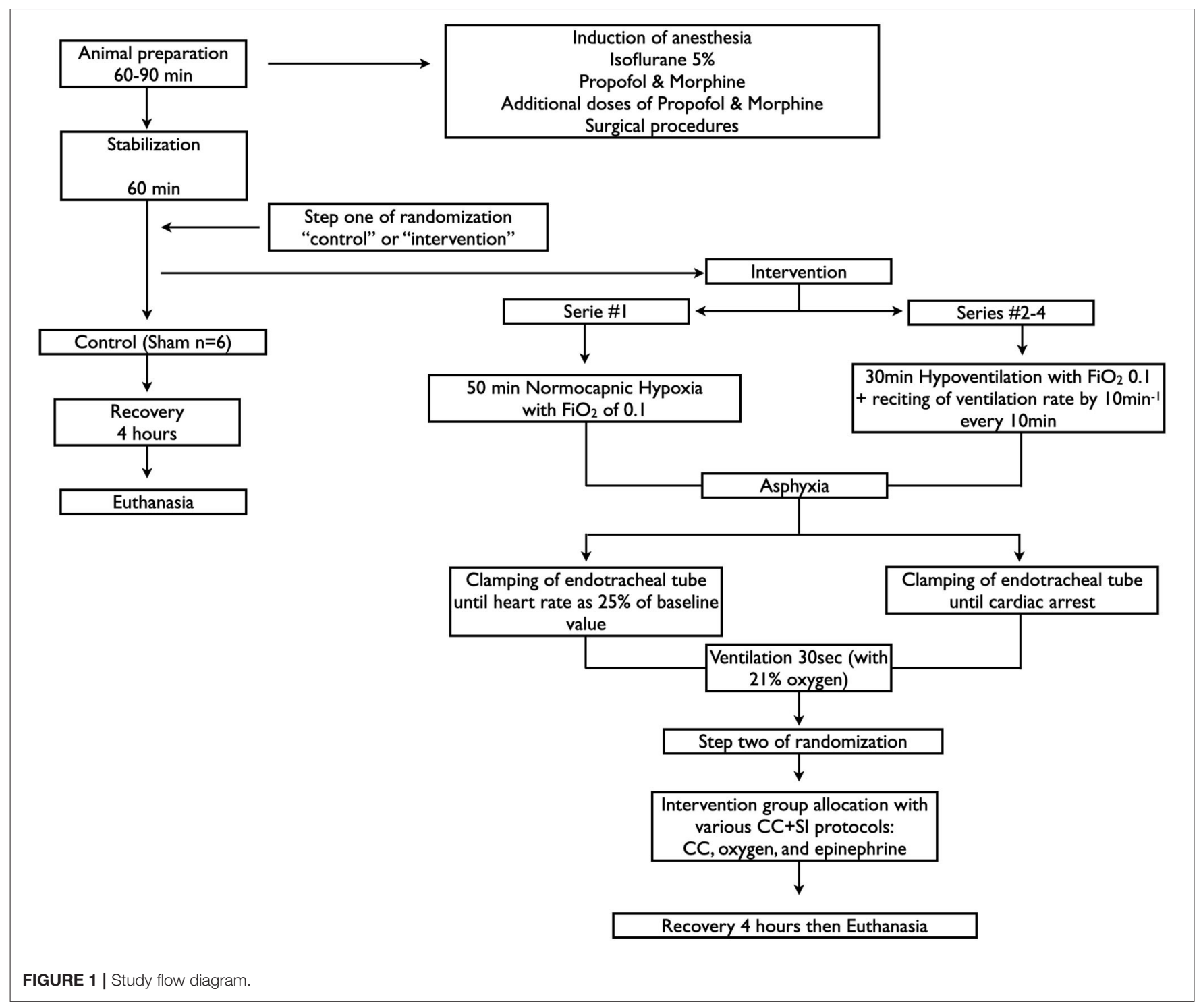

Corp., Troy, MI). The sensors were placed on the right forehead of the piglet and secured with wrap and tape. Light shielding was achieved with a slim cap. The Invos Cerebral/Somatic Oximeter Monitor calculates cerebral oxygenation, which is expressed as the percentage of oxygenated hemoglobin (oxygenated hemoglobin/total hemoglobin). Values of regional oxygen saturation are stored every second with a sample rate of 0.13 $\mathrm{Hz}(23)$.

\section{Experimental Protocol}

In all four studies, piglets were randomized into intervention or sham. To reduce selection bias, a two-step randomization process was used. Following surgical instrumentation and stabilization, a subsequently numbered, sealed opaque envelope containing the assignment "sham" or "intervention" was opened (step one) (Figure 1). Piglets randomized to "intervention" underwent both hypoxia and asphyxia, whereas, piglets randomized to "sham" did not. Sham-operated groups received the same surgical protocol, stabilization, and equivalent experimental periods without hypoxia and asphyxia. The piglets that were randomized to "intervention" were exposed to either $30 \mathrm{~min}$ (asystole group, Studies 2-4, (18)) or 50 min (bradycardia group, Study 1 (11)) of normocapnic hypoxia, which was followed by asphyxia. Asphyxia was achieved by disconnecting the ventilator and clamping the endotracheal tube to a pre-set end-point. Study 1: Asphyxia was induced until bradycardia (defined as a decrease in HR to $25 \%$ of baseline) (11). Studies 2-4: Asphyxia was induced until asystole (defined as no audible HR during continuous auscultation and zero carotid artery blood flow) (18-20). PEA was defined as zero HR but with electrical activity on the ECG $(3,4)$. After the end-point was reached, a second subsequently numbered, sealed opaque envelope containing the intervention assignment was opened (step two) (Figure 1). Fifteen seconds after bradycardia or asystole, positive pressure ventilation was 
TABLE 1 | Baseline characteristics.

\begin{tabular}{|c|c|c|c|c|}
\hline & Bradycardia $(n=24)$ & PEA $(n=29)$ & Asystole $(n=13)$ & $p$-value \\
\hline Age (days) & 2.0 (2.0 to 3.0$)$ & 2.0 (1.0 to 3.0$)$ & 2.0 (1.0 to 2.0$)$ & 0.284 \\
\hline Sex (male/female) & $15 / 9$ & $18 / 11$ & $7 / 6$ & 0.894 \\
\hline $\mathrm{paCO}_{2}(\mathrm{mmHg})$ & 40 (36 to 42) & $34(30 \text { to } 35)^{\star}$ & $33(29 \text { to } 35)^{\star}$ & $<0.001$ \\
\hline $\mathrm{pH}$ & 7.37 (7.34 to 7.42$)$ & $7.49(7.46 \text { to } 7.54)^{\star}$ & $7.55(7.47 \text { to } 7.58)^{\star}$ & $<0.001$ \\
\hline $\mathrm{BE}(\mathrm{mmol} / \mathrm{L})$ & $-2(-6$ to 0$)$ & $2(1 \text { to } 4)^{\star}$ & $4(2 \text { to } 5)^{\star}$ & $<0.001$ \\
\hline $\mathrm{HCO}_{3}^{-}(\mathrm{mmol} / \mathrm{L})$ & 24 (21 to 25) & $25(25 \text { to } 27)^{\star}$ & $27(25 \text { to } 29)^{\star}$ & 0.002 \\
\hline Lactate (mmol/L) & $3.6(2.7$ to 4.1$)$ & 3.6 (2.9 to 4.9$)$ & 3.2 (3.0 to 3.5$)$ & 0.280 \\
\hline Heart rate (bpm) & 228 (215 to 248$)$ & $193(173 \text { to } 211)^{*}$ & $186(160 \text { to } 202)^{\star}$ & $<0.001$ \\
\hline Mean arterial pressure $(\mathrm{mmHg})$ & 77 (69 to 87) & $60(56 \text { to } 66)^{\star}$ & $57(53 \text { to } 63)^{*}$ & $<0.001$ \\
\hline Carotid flow (mL/min) & 77 (65 to 90$)$ & $47(40 \text { to } 56)^{\star}$ & $45(33 \text { to } 51)^{\star}$ & $<0.001$ \\
\hline Cerebral oxygenation index (\%) & 41 (39 to 47 ) & $53(48 \text { to } 57)^{\star}$ & $49(42 \text { to } 58)^{\star}$ & $<0.001$ \\
\hline
\end{tabular}

Data are presented as median (IQR); * significantly different from bradycardia group. PEA, pulsless electrical activity.

performed for $30 \mathrm{~s}$ with a Neopuff T-Piece (Fisher \& Paykel, Auckland, New Zealand). The default settings of the experiment were a peak inflating pressure of $30 \mathrm{cmH}_{2} \mathrm{O}$ (except 16 piglets in study 3 , which were randomized to peak inflating pressure of either 10 or $20 \mathrm{~cm} \mathrm{H}_{2} \mathrm{O}$ ), a positive end expiratory pressure (PEEP) of $5 \mathrm{cmH}_{2} \mathrm{O}$, and a gas flow of $8 \mathrm{~L} / \mathrm{min}$. Using the twothumb encircling technique (7), CC was performed at a rate of $90 /$ min (except eight piglets with rate of $120 /$ min in study 1 ) using a metronome by a single operator in all the piglets. CC was started after $30 \mathrm{~s}$ of positive pressure ventilation and $100 \%$ oxygen was commenced $30 \mathrm{~s}$ after start of CC. Epinephrine $(0.02 \mathrm{mg} / \mathrm{kg}$ per dose) was administered intravenously 2 min after the start of positive pressure ventilation, and administered every $3 \mathrm{~min}$ as needed if no ROSC was observed. Epinephrine was administered to a maximum of 4 doses as the maximum resuscitation time was set at $12 \mathrm{~min}$. ROSC was defined as an unassisted HR > $100 \mathrm{bpm}$ for at least $15 \mathrm{~s}$. After ROSC, piglets recovered for $4 \mathrm{~h}$ before euthanasia with an intravenous overdose of phenobarbital (100 mg/kg).

\section{Data Collection and Analysis}

Demographics of study piglets were recorded. Transonic flow probes, HR, and pressure transducer outputs were digitized and recorded with LabChart ${ }^{\circledR}$ programming software (ADInstruments, Houston, TX). Data are presented as mean (standard deviation) for normally distributed continuous variables and median (interquartile range) when the distribution was skewed. The data was tested for normality and compared using one-way ANOVA for comparisons of continuous variables, and $\chi^{2}$ for categorical variables. Post-hoc analysis was performed using Tukey test. Time to ROSC was analyzed with Cox proportional hazards regression by using SAS Proc SURVEYPHREG with stratification by studies, to account for data being combined from different trials. The event was considered as ROSC. Piglets who did not achieve ROSC were considered censored at a maximum of $12 \mathrm{~min}$ (720s) of cardiopulmonary Resuscitation time. The proportionality assumption was violated for bradycardia and asystole groups by assessment of Kaplan-Meier and Log-Log curves. Therefore, three different Cox proportional hazards regression models were created for pairwise comparison of PEA, bradycardia and asystole groups. Both models for PEA and asystole groups, and PEA and bradycardia groups satisfied proportionality assumption; however, since this assumption was violated for bradycardia and asystole groups, the interaction term (cardiac group)*(time to ROSC) was added to the Cox regression model for those 2 groups. Both terms from that model, cardiac group and interaction (cardiac group)*(time to ROSC), were used to estimate the hazard ratio, which was not constant over time. Statistical analyses were performed with SigmaPlot (Systat Sofyware Inc., San Jose) and SAS Ver. 9.4 (SAS Institute Inc., Cary, NC, USA).

\section{RESULTS}

Age and sex were similar among the three experimental groups (bradycardia, asystole, PEA) (Table 1). However, baseline hemodynamic and blood gas values were significantly better in the bradycardia group compared to the PEA and asystole groups (Table 1). These differences were most likely related to the different surgical procedures, experimental set-up, and medication (10). Therefore, we compared changes from baseline for hemodynamic parameters within the same group.

\section{Resuscitation}

The asphyxia time was significantly longer in the PEA and asystole groups compared to the bradycardia group (Table 2). This resulted in significantly lower $\mathrm{pH}$, higher $\mathrm{paCO}_{2}$, and lactate in both groups compared to the bradycardia group (Table 2).

Time to ROSC was significantly shorter in the bradycardia group compared to the asystole and PEA group (Table 2). The rate of ROSC was significantly lower in the PEA group compared to the bradycardia and asystole groups (Table 2). The total number of epinephrine doses and the number of piglets receiving epinephrine were significantly higher in the PEA group compared to the asystole group (Table 2). There was no significant difference in post-ROSC survival between all three groups (Table 2). In Cox regression models the hazard ratios 
TABLE 2 | Characteristics of asphyxia, resuscitation, and survival of asphyxiated piglets.

\begin{tabular}{|c|c|c|c|c|}
\hline & Bradycardia $(n=24)$ & PEA $(n=29)$ & Asystole $(n=13)$ & $p$-value \\
\hline $\mathrm{PaCO}_{2}$ & 67 (58 to 85) & $98(87 \text { to } 113)^{\star}$ & $116(104 \text { to } 122)^{\star}$ & $<0.001$ \\
\hline $\mathrm{pH}$ & 6.91 (6.83 to 6.98$)$ & $6.59(6.50 \text { to } 6.67)^{\star}$ & $6.50(6.50 \text { to } 6.54)^{\star}$ & $<0.001$ \\
\hline $\mathrm{BE}(\mathrm{mmol} / \mathrm{L})$ & $-20(-22$ to -16$)$ & $-27(-30 \text { to }-26)^{\star}$ & $-30(-30 \text { to }-28)^{\star}$ & $<0.001$ \\
\hline Lactate (mmol/L) & $13.0(11.7$ to 14.1$)$ & $15.8(14.3 \text { to } 17.1)^{\star}$ & $15.7(14.7 \text { to } 16.2)^{\star}$ & $<0.001$ \\
\hline Asphyxiation time (s) & 112 (69 to 180$)$ & $475(221 \text { to } 589)^{*}$ & $332(260 \text { to } 460)^{\star}$ & $<0.001$ \\
\hline ROSC time (s) & 35 (28 to 68) & $120(80 \text { to } 175)^{\star}$ & $69(60$ to 111$)$ & $<0.001$ \\
\hline Number of piglets with ROSC & $20(83 \%)$ & $16(55 \%)^{\star \#}$ & $13(100 \%)$ & $<0.01$ \\
\hline Total number of Epi dose & 0 (0 to 3$)$ & $1(0 \text { to } 4)^{\#}$ & $0(0$ to 0.5$)$ & 0.004 \\
\hline Number of piglets receiving Epi & $10(42 \%)$ & $20(69 \%)^{\#}$ & $3(23 \%)$ & $<0.01$ \\
\hline Overall survival after resuscitation & 20 (100\%) & $16(100 \%)$ & $12(92 \%)$ & 0.26 \\
\hline
\end{tabular}

Data are presented as median (IQR); Epi, epinephrine; *Significantly different from bradycardia group; \#Significantly different from asystole group. PEA, pulsless electrical activity.

between subjects from different cardiac groups are presented in Table 3. For bradycardia and asystole groups hazard ratio depended on time. As time increased, the hazard ratio increased as well.

\section{Changes in Hemodynamic Parameters}

Hemodynamic changes throughout the experiment are summarized in Figure 2. Heart rate significantly decreased at the end of asphyxia compared to baseline in all three groups, and returned to baseline after ROSC, with significantly higher $\mathrm{HR}$ in the PEA and asystole groups than that of bradycardia group (Figure 2).

The mean arterial blood pressure decreased significantly from baseline values after asphyxia (Figure 2), returned to baseline after ROSC, and then gradually decreased over the $4 \mathrm{~h}$ observation period. Compared to baseline, all groups had a significantly lower mean arterial pressure at the end of the $4 \mathrm{~h}$ observation period (Figure 2). The mean arterial pressure in the PEA group was significantly lower compared to the bradycardia group (Figure 2).

At the end of asphyxia, the CABF significantly decreased in all three groups compared to baseline (Figure 2). At the end of the $4 \mathrm{~h}$ observation period, the CABF remained significantly lower compared to baseline. Of note, the CABF was significantly lower in the PEA and asystole groups compared to the bradycardia group at the end of the $4 \mathrm{~h}$ observation period [36(30)\%, $36(30) \%$, and $75(35) \%$ of baseline value for PEA, asystole and bradycardia group, respectively] (Figure 2). Consequently, the cerebral oxygenation in the PEA and asystole groups were also significantly lower compared to the bradycardia group at the end of the $4 \mathrm{~h}$ observation period (Figure 2).

\section{Changes in Blood Gas}

The $\mathrm{pH}$ in all groups significantly decreased from baseline values after asphyxia (Table 4). It improved after ROSC and gradually increased throughout the $4 \mathrm{~h}$ observation period. At the end of the $4 \mathrm{~h}$ observation period, the $\mathrm{pH}$ in the bradycardia group was similar compared to baseline, whereas the $\mathrm{pH}$ in the PEA and asystole groups remained significantly lower than their baseline
(Table 4). There were similar patterns of changes in base excess, bicarbonate, and lactate throughout the experiment (Table 4).

\section{DISCUSSION}

There is limited evidence about management of different cardiac arrest rhythms during neonatal resuscitation and their outcome (4-6, 24). While healthcare providers routinely recognize bradycardia and asystole in the delivery room, the detection of PEA rhythms has only been described recently (4). This is the first study that investigated the influence of different cardiac arrest rhythms on resuscitation outcomes and recovery in asphyxiated piglets resuscitated by different $\mathrm{CC}+\mathrm{SI}$ approaches. Compared to both the bradycardia and asystole groups, the PEA group required a longer resuscitation time and had less animals achieving ROSC and overall survival. Furthermore, epinephrine was used more often in the PEA group compared to the two other experimental groups. Our results also demonstrated that the hemodynamic recovery of the bradycardia group was better than the PEA and asystole groups.

Recent animal studies $(9,11,25,26)$ and clinical observations (12) have indicated that CC+SI significantly improves ROSC, tidal volume delivery and minute ventilation, as well as global and regional hemodynamics compared to conventional 3:1 $\mathrm{C}: \mathrm{V}$. Currently, a large cluster randomized trial comparing $\mathrm{CC}+\mathrm{SI}$ with 3:1 C:V in newborn infants is ongoing (SURV1VE) (13). Therefore, our study focused on the effect of CC+SI on resuscitation outcomes and hemodynamic recovery in asphyxiated piglets with different heart rhythms (bradycardia, $\mathrm{PEA}$, or asystole). As indicated by lower $\mathrm{pH}$, higher base excess and lactate levels, the hypoxia/asphyxia stress in both PEA and asystole groups were more severe than in the bradycardia group. Consequently, a prolonged time to ROSC, particularly in the PEA group, was observed compared to the bradycardia group. In a neonatal lamb asphyxia model lactate levels were also higher in animals with no ROSC (27). Further, the $\mathrm{pH}$ of these two groups remained lower throughout the experiment whereas the $\mathrm{pH}$ in the bradycardia group returned to baseline by the end of the $4 \mathrm{~h}$ observation period. These observations were similar to that reported following resuscitation of newborn infants with 
TABLE 3 | Cox Regression estimates of hazard ratios.

\begin{tabular}{llcccc}
\hline Model & & Estimate & SE & p-value & Univariate hazard ratio (95\% Cl) \\
\hline Asystole vs. PEA & & 1.51 & 0.34 & $<0.0001$ & $4.53(2.29,8.97)$ \\
Bradycardia vs. PEA & & 1.12 & 0.33 & 0.002 & $3.05(1.58,5.91)$ \\
Bradycardia vs. Asystole & Bradycardia vs. Asystole & 1.96 & 0.71 & 0.01 & $7.07(1.65,30.34)$ \\
& Cardiac group*time to ROSC (interaction) & 0.03 & 0.01 & 0.02 & $1.03(1.003,1.05)$
\end{tabular}
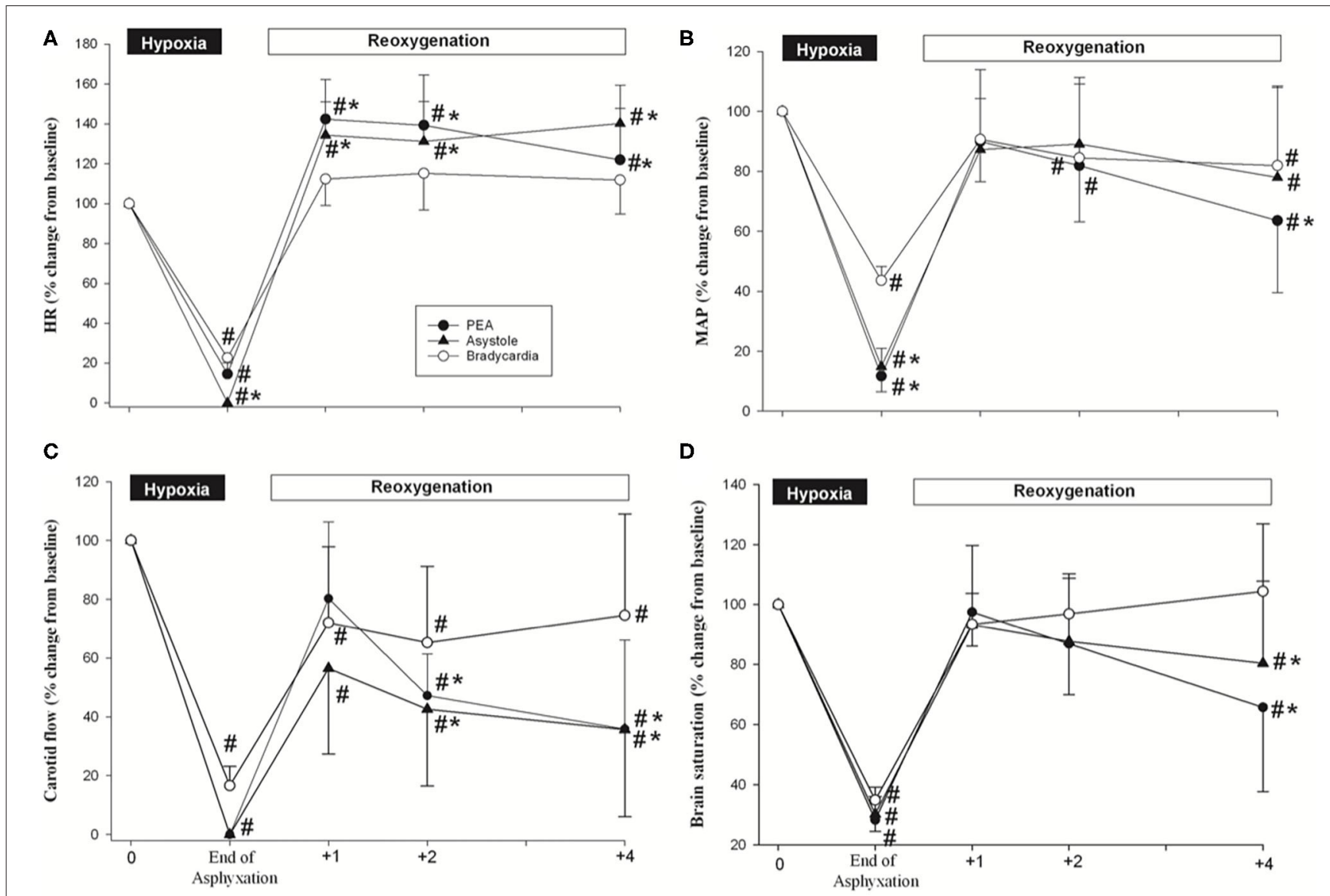

\section{Time (h)}

FIGURE 2 | Percentage changes from normoxic baseline in (A) heart rate, (B) mean arterial pressure (MAP), (C) carotid blood flow, and (D) brain saturation in bradycardia (०), PEA $(\bullet)$, and asystole $(\mathbf{\Lambda})$ groups during hypoxia/asphyxia and after resuscitation. Each point represents mean \pm SD. ${ }^{*} p<0.05$, significantly different from bradycardia group; $\# p<0.05$, significantly different from its own baseline value.

bradycardia or asystole (17). In that study, asystole was associated with a lower cord blood $\mathrm{pH}$ and a lower $\mathrm{pH} 1 \mathrm{~h}$ after birth, and infants required significantly more intubations and CCs (17). Although bradycardia has been associated with higher survival rates compared to asystole/PEA in pediatric patients (28), we did not observe any difference in survival rate after the $4 \mathrm{~h}$ observation period; however, this is likely due to the relatively short period of recovery.

Although the PEA and asystole groups had similarities in the asphyxia duration and blood gas parameters, the ROSC time was about twice of that of the asystole group. Furthermore, there were significantly more piglets that required epinephrine in the PEA group compared to the asystole group, and the number of doses was also significantly greater. Consequently, only 55\% of piglets with PEA achieved ROSC. Given the observational nature of this study, our data did not investigate any underlying mechanisms contributing to PEA. Zheng et al. (29) observed a better resuscitation outcome in adult cardiac arrest patients presenting with asystole than those with PEA. They postulated that the pumping activity of the heart was limited in PEA patients during resuscitation due to dissociation of the pumping mechanism, whereas the insufficiency of cardiac output in 
TABLE 4 | Blood gas at baseline, after asphyxiation, and $4 \mathrm{~h}$ of reoxygenation.

\begin{tabular}{|c|c|c|c|c|}
\hline & Bradycardia & With PEA & Asystole & $\mathrm{p}$-value \\
\hline \multicolumn{5}{|l|}{ pH } \\
\hline Baseline & $7.37(0.07)$ & $7.49(0.06)^{\star}$ & $7.53(0.07)^{\star}$ & $<0.001$ \\
\hline After asphyxiation & $6.90(0.11)^{\#}$ & $6.60(0.09)^{* \#}$ & $6.53(0.05)^{\star \#}$ & $<0.001$ \\
\hline $4 \mathrm{~h}$ after reoxygenation & $7.33(0.07)$ & $7.29(0.10)^{\#}$ & $7.36(0.13)^{\#}$ & 0.082 \\
\hline \multicolumn{5}{|l|}{$\mathrm{PaCO}_{2}(\mathrm{mmHg})$} \\
\hline Baseline & $42(6)$ & $34(4)^{\star}$ & $32(2)^{\star}$ & $<0.001$ \\
\hline After asphyxiation & $71(19)^{\#}$ & $100(18)^{\star \#}$ & $114(11)^{\star \#}$ & $<0.001$ \\
\hline $4 \mathrm{~h}$ after reoxygenation & $41(6)$ & $37(6)^{\star}$ & $36(6)^{\star}$ & $<0.001$ \\
\hline \multicolumn{5}{|l|}{ Base excess (mmol/L) } \\
\hline Baseline & $-2(4)$ & $2(3)^{\star}$ & $4(4)^{\star}$ & $<0.001$ \\
\hline After asphyxiation & $-19(5)^{\#}$ & $-28(2)^{\star \#}$ & $-29(2)^{\star \#}$ & $<0.001$ \\
\hline $4 \mathrm{~h}$ after reoxygenation & $-4(2)$ & $-8(5)^{\star \#}$ & $-5(6)^{\#}$ & 0.005 \\
\hline \multicolumn{5}{|l|}{$\mathrm{HCO}_{3}(\mathrm{mmol} / \mathrm{L})$} \\
\hline Baseline & $24(3)$ & $26(2)^{\star}$ & $27(3)^{\star}$ & 0.002 \\
\hline After asphyxiation & $12(2)^{\#}$ & $9(3)^{\star \#}$ & $9(4)^{\star \#}$ & $<0.001$ \\
\hline $4 \mathrm{~h}$ after reoxygenation & $21(3)$ & $18(4)^{\star \#}$ & $20(4)^{\#}$ & 0.014 \\
\hline \multicolumn{5}{|l|}{ Lactate (mmol/L) } \\
\hline Baseline & $3.7(1.0)$ & $4.1(0.9)$ & $3.6(0.7)$ & 0.353 \\
\hline After asphyxiation & $12.9(2.1)^{\#}$ & $15.6(2.2)^{\star \#}$ & $15.4(2.7)^{\star \#}$ & $<0.001$ \\
\hline $4 \mathrm{~h}$ after reoxygenation & $4.1(2.4)$ & $5.7(3.3)^{\#}$ & $4.5(2.6)$ & 0.121 \\
\hline
\end{tabular}

Data are presented as mean (SD); *Significantly different from bradycardia group, \#Significantly different from baseline values. PEA, pulsless electrical activity.

asystole patients was only due to lack of electrical pacing. Further studies are needed to test this hypothesis in our animal studies. Collectively, our data indicates that heart rhythm influences resuscitation outcome and recovery following CC+SI.

Sobotka et al. (30) described that HR might be a weak indicator for assessing circulatory status in asphyxiated newborns as even with a mean $\mathrm{HR}$ of $72 \pm 7 / \mathrm{min}$ was associated with zero cerebral blood flow in their animal model. In a clinical case series of four neonatal patients, PEA was observed with an electrical HR above 60/min (as displayed on ECG) but either no palpable pulses or a HR assessed by auscultation below 60/min (6). All four patients died after extensive resuscitation, supporting the speculation that PEA is a critical problem in the delivery room. If the HR is only assessed by ECG, this could be misleading and may delay initiation of CCs. A new algorithm approach was described in Luong et al. (6), suggesting using auscultation or palpation as well as ECG and a pulse oximeter for the assessment of a newborn. Doppler ultrasound would be a good alternative to detect PEA, although it is not routinely used (3). Newborns typically present with bradycardia before descending into asystole (28). Therefore, it is necessary to combine ECG monitoring with clinical findings (pulse palpation, auscultation) or pulse oximetry (no pulsatile waveform) for identification of PEA (3). Further studies are needed to assess the prevalence and influence of different heart rhythms in the delivery room.

\section{Limitations}

Our asphyxia model uses piglets that have already undergone the fetal to neonatal transition, and piglets were sedated/anesthetized. Furthermore, our model requires piglets to be intubated with a tightly sealed endotracheal tube to prevent any endotracheal tube leak; this may not occur in the delivery room as mask ventilation is frequently used, in addition to the presence of leak in endotracheally intubated infants. Our experimental protocol differed slightly to the current resuscitation guidelines: we used $100 \%$ oxygen after 30 s of CC and administered epinephrine $90 \mathrm{~s}$ after CC with a frequency of one dose every $3 \mathrm{~min}$. Furthermore, our baseline values were different between the groups, which are likely due to the slight variability in surgical procedures and anesthetic drugs used. Although we conformed to the 3 R's of animal studies (Replacement, Reduction, and Refinement), we understand that using results of previous studies with different piglet models and different numbers of piglets to perform a secondary analysis also may pose a limitation to data interpretation which were studied using the changes from respective baselines. Further, we acknowledge that as in all animal studies, it may not be possible to translate the results necessarily to human newborns.

\section{CONCLUSIONS}

Our study indicates that heart rhythm before resuscitation (bradycardia, asystole, and PEA) influences the time to ROSC, epinephrine administration, and hemodynamic recovery after resuscitation. Evaluation of heart rhythms in the delivery room and their correlation with resuscitation outcome warrants further investigation.

\section{DATA AVAILABILITY STATEMENT}

The raw data supporting the conclusions of this article will be made available by the authors, without undue reservation.

\section{ETHICS STATEMENT}

The animal study was reviewed and approved by Animal Care and Use Committee (Health Sciences), University of Alberta.

\section{AUTHOR CONTRIBUTIONS}

GS conceptualized and designed the study. MW drafted the initial manuscript. MW, T-FL, and GS designed the data collection instruments, collected data, carried out the initial analyses, and reviewed and revised the manuscript. P-YC, MY, VV, and MO'R helped interpreting the results and reviewed and revised the manuscript. All authors approved the final manuscript as submitted and agree to be accountable for all aspects of the work.

\section{FUNDING}

We would like to thank the public for donating money to our funding agencies: GS is a recipient of the Heart and Stroke Foundation/University of Alberta Professorship of Neonatal Resuscitation, a National New Investigator of the Heart and Stroke Foundation Canada and an Alberta New Investigator 
of the Heart and Stroke Foundation Alberta. We would like to acknowledge the Neonatal Resuscitation Program Canada Grant Program within the Canadian Society of Pediatrics, a Grant from the SickKids Foundation in partnership with the Canadian Institutes of Health Research [CIHR-Institute of
Human Development, Child and Youth Health (IHDCYH)], New Investigator Research Grant Program (Grant number-No. NI17-033). This research has been facilitated by the Women and Children's Health Research Institute through the generous support of the Stollery Children's Hospital Foundation.

\section{REFERENCES}

1. Wyckoff MH, Wyllie J, Aziz K, de Almeida MF, Fabres J, Fawke J, et al. neonatal life support, neonatal life support: 2020 International Consensus on Cardiopulmonary Resuscitation and Emergency Cardiovascular Care Science With Treatment Recommendations. Circulation. (2020) 142:S185S221. doi: 10.1016/j.resuscitation.2020.09.015

2. Agrawal V, Lakshminrusimha S, Chandrasekharan P. Chest compressions for bradycardia during neonatal resuscitation-do we have evidence? Children (Basel). (2019) 6:119. doi: 10.3390/children6110119

3. Luong DH, Cheung PY, O’Reilly M, Lee TF, Schmolzer GM. Electrocardiography vs. auscultation to assess heart rate during cardiac arrest with pulseless electrical activity in newborn infants. Front Pediatr. (2018) 6:366. doi: 10.3389/fped.2018.00366

4. Pasquin MP, Cheung PY, Patel S, Lu M, Lee TF, Wagner M, et al. Comparison of different compression to ventilation ratios (2: 1, 3: 1, and 4:1) during cardiopulmonary resuscitation in a porcine model of neonatal asphyxia. Neonatology. (2018) 114:37-45. doi: 10.1159/000487988

5. Sillers L, Handley SC, James JR, Foglia EE. pulseless electrical activity complicating neonatal resuscitation. Neonatology. (2018) 115:95-8. doi: $10.1159 / 000493357$

6. Luong D, Cheung PY, Barrington KJ, Davis PG, Unrau J, Dakshinamurti $\mathrm{S}$, et al. Cardiac arrest with pulseless electrical activity rhythm in newborn infants: a case series. Arch Dis Child Fetal Neonatal Ed. (2019) 104:F572-4. doi: 10.1136/archdischild-2018-316087

7. Wyckoff MH, Aziz K, Escobedo MB, Kapadia VS, Kattwinkel J, Perlman JM, et al. Part 13: neonatal resuscitation: 2015 American Heart Association Guidelines Update for Cardiopulmonary Resuscitation and Emergency Cardiovascular Care. Circulation. (2015) 132:S543-60. doi: 10.1161/CIR.0000000000000267

8. Perlman JM, Wyllie J, Kattwinkel J, Wyckoff MH, Aziz K, Guinsburg R, et al. Neonatal Resuscitation Chapter, Part 7: Neonatal Resuscitation: 2015 International Consensus on Cardiopulmonary Resuscitation and Emergency Cardiovascular Care Science With Treatment Recommendations. Circulation. (2015) 132:S204-41. doi: 10.1161/CIR.0000000000000276

9. Schmolzer GM, O’Reilly M, Labossiere J, Lee TF, Cowan S, Qin S, et al. Cardiopulmonary resuscitation with chest compressions during sustained inflations: a new technique of neonatal resuscitation that improves recovery and survival in a neonatal porcine model. Circulation. (2013) 128:2495-503. doi: 10.1161/CIRCULATIONAHA.113.002289

10. Li ES, Cheung PY, Lee TF, Lu M, O’Reilly M, Schmolzer GM. Return of spontaneous circulation is not affected by different chest compression rates superimposed with sustained inflations during cardiopulmonary resuscitation in newborn piglets. PLoS One. (2016) 11:e0157249. doi: 10.1371/journal.pone.0157249

11. Li ES, Gorens I, Cheung PY, Lee TF, Lu M, O’Reilly M, et al. Chest compressions during sustained inflations improve recovery when compared to a 3:1 compression:ventilation ratio during cardiopulmonary resuscitation in a neonatal porcine model of asphyxia. Neonatology. (2017) 112:337-46. doi: $10.1159 / 000477998$

12. Schmolzer GM, O’Reily M, Fray C, van Os S, Cheung PY. Chest compression during sustained inflation versus 3:1 chest compression:ventilation ratio during neonatal cardiopulmonary resuscitation: a randomised feasibility trial. Arch Dis Child Fetal Neonatal Ed. (2018) 103:F455-F60. doi: 10.1136/archdischild-2017-313037

13. Schmolzer GM, Pichler G, Solevåg AL, Fray C, van Os S, Cheung PY. The SURV1VE trial-sustained inflation and chest compression versus 3:1 chest

compression-to-ventilation ratio during cardiopulmonary resuscitation of asphyxiated newborns: study protocol for a cluster randomized controlled trial. Trials. (2019) 20:139. doi: 10.1186/s13063-019-3240-8

14. Schmolzer GM, O'Reilly M, Labossiere J, Lee TF, Cowan S, Nicoll J, et al. 3:1 compression to ventilation ratio versus continuous chest compression with asynchronous ventilation in a porcine model of neonatal resuscitation. Resuscitation. (2014) 85:270-5. doi: 10.1016/j.resuscitation.2013.10.011

15. Andersen LW, Holmberg MJ, Berg KM, Donnino MW, Granfeldt A. In-hospital cardiac arrest: a review. JAMA. (2019) 321:1200-10. doi: 10.1001/jama.2019.1696

16. Meert KL, Telford R, Holubkov R, Slomine BS, Christensen JR, Dean JM, et al. Therapeutic hypothermia after pediatric cardiac arrest trial, pediatric outof-hospital cardiac arrest characteristics and their association with survival and neurobehavioral outcome. Pediatr Crit Care Med. (2016) 17:e543-e50. doi: 10.1097/PCC.0000000000000969

17. Kumar VH, Skrobacz A, Ma C. Impact of bradycardia or asystole on neonatal cardiopulmonary resuscitation at birth. Pediatr Int. (2017) 59:891-7. doi: $10.1111 /$ ped.13310

18. Mustofa J, Cheung PY, Patel S, Lee TF, Lu M, Pasquin MP, et al. Effects of different durations of sustained inflation during cardiopulmonary resuscitation on return of spontaneous circulation and hemodynamic recovery in severely asphyxiated piglets. Resuscitation. (2018) 129:82-9. doi: 10.1016/j.resuscitation.2018.06.013

19. Shim GH, Kim SY, Cheung PY, Lee TF, O’Reilly M, Schmolzer GM. Effects of sustained inflation pressure during neonatal cardiopulmonary resuscitation of asphyxiated piglets. PLoS One. (2020) 15:e0228693. doi: 10.1371/journal.pone.0228693

20. Hidalgo CG, Solevag AL, Kim SY, Shim GH, Cheung PY, Lee TF, et al. Sustained inflation with $21 \%$ versus $100 \%$ oxygen during cardiopulmonary resuscitation of asphyxiated newborn piglets - a randomized controlled animal study. Resuscitation. (2020) 155:39-47. doi: 10.1016/j.resuscitation.2020.07.015

21. Kilkenny C, Browne WJ, Cuthill IC, Emerson M, Altman DG. Improving bioscience research reporting: the ARRIVE guidelines for reporting animal research. PLoS Biol. (2010) 8:e1000412. doi: 10.1371/journal.pbio.1000412

22. Cheung P, Gill RS, Bigam DL. A swine model of neonatal asphyxia. J Vis Exp. (2011) e3166. doi: 10.3791/3166

23. Pichler G, Binder C, Avian A, Beckenbach E, Schmolzer GM, Urlesberger B. Reference ranges for regional cerebral tissue oxygen saturation and fractional oxygen extraction in neonates during immediate transition after birth. $J$ Pediatr. (2013) 163:1558-63. doi: 10.1016/j.jpeds.2013.07.007

24. Mehta C, Brady W. Pulseless electrical activity in cardiac arrest: electrocardiographic presentations and management considerations based on the electrocardiogram. Am J Emerg Med. (2012) 30:236-9. doi: 10.1016/j.ajem.2010.08.017

25. Li ES, Cheung PY, O'Reilly M, Schmolzer GM. Change in tidal volume during cardiopulmonary resuscitation in newborn piglets. Arch Dis Child Fetal Neonatal Ed. (2015) 100:F530-3. doi: 10.1136/archdischild-2015-308363

26. Solevag AL, Lee TF, Lu M, Schmolzer GM, Cheung PY. Tidal volume delivery during continuous chest compressions and sustained inflation. Arch Dis Child Fetal Neonatal Ed. (2017) 102:F85-F7. doi: 10.1136/archdischild-2016-311043

27. Vali $\mathrm{P}$, Chandrasekharan $\mathrm{P}$, Rawat $\mathrm{M}$, Gugino S, Koenigsknecht $\mathrm{C}$, Helman J, et al. Hemodynamics and gas exchange during chest compressions in neonatal resuscitation. PLoS One. (2017) 12:e0176478. doi: 10.1371/journal.pone.0176478

28. Donoghue A, Berg RA, Hazinski MF, Praestgaard AH, Roberts K, Nadkarni VM, et al. Cardiopulmonary resuscitation for bradycardia with poor 
perfusion versus pulseless cardiac arrest. Pediatrics. (2009) 124:1541-8. doi: 10.1542/peds.2009-0727

29. Zheng R, Luo S, Liao J, Liu Z, Xu J, Zhan H, et al. Conversion to shockable rhythms is associated with better outcomes in out-of-hospital cardiac arrest patients with initial asystole but not in those with pulseless electrical activity. Resuscitation. (2016) 107:88-93. doi: 10.1016/j.resuscitation.2016. 08.008

30. Sobotka KS, Polglase GR, Schmolzer GM, Davis PG, Klingenberg C, Hooper SB. Effects of chest compressions on cardiovascular and cerebral hemodynamics in asphyxiated near-term lambs. Pediatr Res. (2015) 78:395400. doi: $10.1038 /$ pr.2015.117
Conflict of Interest: The authors declare that the research was conducted in the absence of any commercial or financial relationships that could be construed as a potential conflict of interest.

Copyright (๑) 2021 Wagner, Cheung, Yaskina, Lee, Vieth, O'Reilly and Schmölzer. This is an open-access article distributed under the terms of the Creative Commons Attribution License (CC BY). The use, distribution or reproduction in other forums is permitted, provided the original author(s) and the copyright owner(s) are credited and that the original publication in this journal is cited, in accordance with accepted academic practice. No use, distribution or reproduction is permitted which does not comply with these terms. 